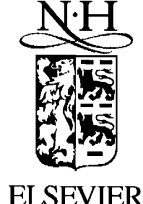

\title{
Effect of La doping on magnetotransport and magnetic properties of double perovskite $\mathrm{Sr}_{2} \mathrm{FeMoO}_{6}$ system
}

\author{
G. Narsinga Rao ${ }^{\mathrm{a}}$, Saibal Roy ${ }^{\mathrm{a}, 1}$, Chung-Yuan Mou ${ }^{\mathrm{b}}$, J.W. Chen ${ }^{\mathrm{a}, *}$ \\ ${ }^{a}$ Department of Physics, National Taiwan University, Taipei, Taiwan, ROC \\ ${ }^{\mathrm{b}}$ Department of Chemistry, National Taiwan University, Taipei, Taiwan, ROC
}

Received 26 November 2004; received in revised form 18 April 2005

Available online 25 May 2005

\begin{abstract}
We have investigated the magnetotransport and magnetic properties on polycrystalline samples of $\mathrm{Sr}_{2-x} \mathrm{La}_{x} \mathrm{FeMoO}_{6}$ $(x=0,0.2,0.4,0.6,0.8$ and 1.0). The magnitude of intergrain tunneling magnetoresistance with low magnetic field of $0.88 \mathrm{~T}$ for $x=0.2$ and 0.4 samples are as large as $5 \%$ and $7 \%$ at room temperature and $13 \%$ and $10 \%$ at $10 \mathrm{~K}$, respectively. The increase of coercivity $\left(H_{\mathrm{c}}\right)$, ratio of remanent magnetization with respect to saturation magnetization $\left(M_{\mathrm{r}} / M_{\mathrm{s}}\right)$, high saturation fields, and reduction of the saturation magnetization indicate that random disorder of spin orientation is mainly responsible for enhancement of the low-field magnetoresistance for samples with $x \leqslant 0.4$. Whereas rapid drop of $H_{\mathrm{c}}, M_{\mathrm{r}} / M_{\mathrm{s}}, M_{\mathrm{r}}$, and saturation fields for samples with $x>0.4$ signifies the growth of antiphase boundary, which gives rise to lower values of low-field MR.
\end{abstract}

(C) 2005 Elsevier B.V. All rights reserved.

PACS: 75.30.Cr; 75.47.Gk

Keywords: Double perovskites; Magnetoresistance; Magnetic properties; Electron doping

\section{Introduction}

Prevailing class of compounds [1,2] which show colossal magnetoresistance (CMR) are of the type

\footnotetext{
*Corresponding author. Tel.: + 886233665172 ; fax: +886223929578 .

E-mail addresses: sroy@tyndall.ie (S. Roy), jwchen@phys.ntu.edu.tw (J.W. Chen).

${ }^{1}$ Present address: Tyndall National Institute, Cork, Ireland.
}

$\mathrm{R}_{1-x} \mathrm{~A}_{x} \mathrm{MnO}_{3}$ where $\mathrm{R}=\mathrm{La}, \mathrm{Pr}, \mathrm{Nd}, \mathrm{Sm}$ and $\mathrm{A}=\mathrm{Ca}, \mathrm{Sr}, \mathrm{Ba}, \mathrm{Pb}$. The $\mathrm{Mn}^{3+} / \mathrm{Mn}^{4+}$ mixed valance states are mainly responsible for the observed magentotransport properties arising from double exchange mechanism [3]. A very large negative magnetoresistance (MR) has been observed at relatively higher magnetic fields at low temperature, but with increasing temperature MR ratio decreases rapidly. Recent interest has been focused on the magnetotransport properties of the 
ordered double perovskite $\mathrm{A}_{2} \mathrm{BB}^{\prime} \mathrm{O}_{6}(\mathrm{~A}=\mathrm{Ca}, \mathrm{Sr}$, $\mathrm{Ba} ; \mathrm{B}=3 \mathrm{~d}$ transition metal, and $\mathrm{B}^{\prime}=\mathrm{Mo}, \mathrm{W}$, $\mathrm{Re})$ due to large MR ratio at room temperature, weak temperature dependence of MR ratio and high Curie temperature $(>400 \mathrm{~K})$ [4-13]. The ordered double perovskite polycrystalline $\mathrm{Sr}_{2} \mathrm{Fe}$ $\mathrm{MoO}_{6}$ was recently found to exhibit large spin polarized intergrain tunneling magnetoresistance of about $10 \%$ at room temperature in the magnetic field of $7 \mathrm{~T}$ [4]. For practical applications such as magnetic field sensors, magnetic storage devices, [14-17], a high MR ratio in low field and at room temperature would be highly desirable.

In $\mathrm{Sr}_{2} \mathrm{FeMoO}_{6}$ the crystal symmetry is known as cubic [6-9] or tetragonal [10,11] with regular arrangement of the alternating $\mathrm{FeO}_{6}$ and $\mathrm{MoO}_{6}$ octahedra. The experimental evidence of ferrimagnetic order as well as band structure results in establishing an antiferromagnetic coupling between $\mathrm{Fe}$ and $\mathrm{Mo}$ atoms having the $\mathrm{Fe}-\mathrm{O}-\mathrm{Mo}$ bond angle of $180^{\circ}$. The density functional calculations for electronic structure $[4,11,18]$ suggest the majority localized up spin electrons of $\mathrm{Fe}^{3+}\left(3 \mathrm{~d}^{5} ; \mathrm{t}_{2 \mathrm{~g}}^{3} \mathrm{e}_{\mathrm{g}}^{2}\right.$; spin $\left.=\frac{5}{2}\right)$ give rise to high spin polarization and the minority itinerant down spin electrons of $\mathrm{Mo}^{5+}\left(4 \mathrm{~d}^{1} ; \mathrm{t}_{2 \mathrm{~g}}^{1}\right.$; spin $\left.=\frac{1}{2}\right)$ are occupied in conduction band. One can expect substitution of divalent alkaline earth by a trivalent La will reduce the mean size of the $\mathrm{A}$ site cations and produce an expansion of the mean radius of the atomic species at $\left(\mathrm{B}, \mathrm{B}^{\prime}\right)$ sites of the $\left(\mathrm{A}_{2-x} \mathrm{~A}_{x}^{\prime}\right) \mathrm{BB}^{\prime} \mathrm{O}_{6}$. Some recent [19-22] experiments have shown that the enhancement of Curie temperature by partial substitution of $\mathrm{La}^{3+}$ for $\mathrm{Sr}^{2+}$ in $\mathrm{Sr}_{2} \mathrm{FeMoO}_{6}$. The size mismatch significantly alters the bond lengths and bond angles for $\mathrm{Fe}-\mathrm{O}-\mathrm{Mo}$ and $\mathrm{Mo}-\mathrm{Mo}$, hence the magnetic interaction between $\mathrm{Fe}$ and Mo atoms, which in turn affects the electronic and magnetic properties. In this paper, we have investigated La doping effect on the magnetotransport and magnetic properties in double perovskite $\mathrm{Sr}_{2-x} \mathrm{La}_{x} \mathrm{FeMoO}_{6}$.

\section{Experimental}

$\mathrm{Sr}_{2-x} \mathrm{La}_{x} \mathrm{FeMoO}_{6}(x=0,0.2,0.4,0.6,0.8$ and 1.0) polycrystalline samples have been prepared from high purity powders of $\mathrm{SrCO}_{3}, \mathrm{La}_{2} \mathrm{O}_{3}, \mathrm{Fe}_{2} \mathrm{O}_{3}$ and $\mathrm{MoO}_{6}(99.99 \%$ purity, Johnson Mathews) taken in proper stoichiometric ratio. Powders are ground in a mortar in acetone medium for homogeneous mixing and calcined at $900{ }^{\circ} \mathrm{C}$ for $6 \mathrm{~h}$ in air. The calcined powders are reground and recalcined at $1000^{\circ} \mathrm{C}$ for $6 \mathrm{~h}$ in air. The final powders are ground again and then cold-pressed into pellets with $5000 \mathrm{lb}$ pressure for $5 \mathrm{~min}$ and sintered at $1200{ }^{\circ} \mathrm{C}$ for $3 \mathrm{~h}$ in flowing $10 \% \mathrm{H}_{2} / \mathrm{N}_{2}$ atmosphere. Room temperature X-ray powder diffraction patterns were obtained using SCINTAG (X1) and $\mathrm{Cu}-\mathrm{K} \alpha$ radiation. The grain size has been estimated from the scanning electron micrographs using Hitachi S-3000. The DC resistance and magnetoresistance have been measured by standard four probe technique using close cycle helium cryostat from 10 to $300 \mathrm{~K}$ (CTI Cryogenics, USA) placed in a conventional water cooled electromagnet with a field from -0.88 to $+0.88 \mathrm{~T}$. The DC magnetization has been measured in commercial SQUID magnetometer (CONDUCTUS, USA) with various fields from -5 to $+5 \mathrm{~T}$.

\section{Results and discussion}

X-ray diffraction patterns of polycrystalline samples of $\mathrm{Sr}_{2-x} \mathrm{La}_{x} \mathrm{FeMoO}_{6}$ with $x=0,0.2,0.6$ and 1.0 are presented in Fig. 1. The insets shows the logarithmic scale of extended reflection around $39.5^{\circ}$ for $x=0$ and 1.0 samples. There is no other distortion in doped and undoped samples. It shows single phase patterns of cubic crystalline structure with space group Fm3m for all the samples. The calculated lattice parameters are listed in Table 1. The considerable cell contraction is observed with $\mathrm{La}$ substitution.

Figs. 2(a) and (b) show scanning electron micrographs of $\mathrm{Sr}_{1.6} \mathrm{La}_{0.4} \mathrm{FeMoO}_{6}$ and SrLaFe$\mathrm{MoO}_{6}$ samples respectively, which are typical micrographs for all the samples. Fig. 3 shows histogram of number of grains with grain diameter for $\mathrm{SrLaFeMoO}_{6}$ which represents a typical histogram for all the samples. We have tried to fit histograms of all the samples with the 


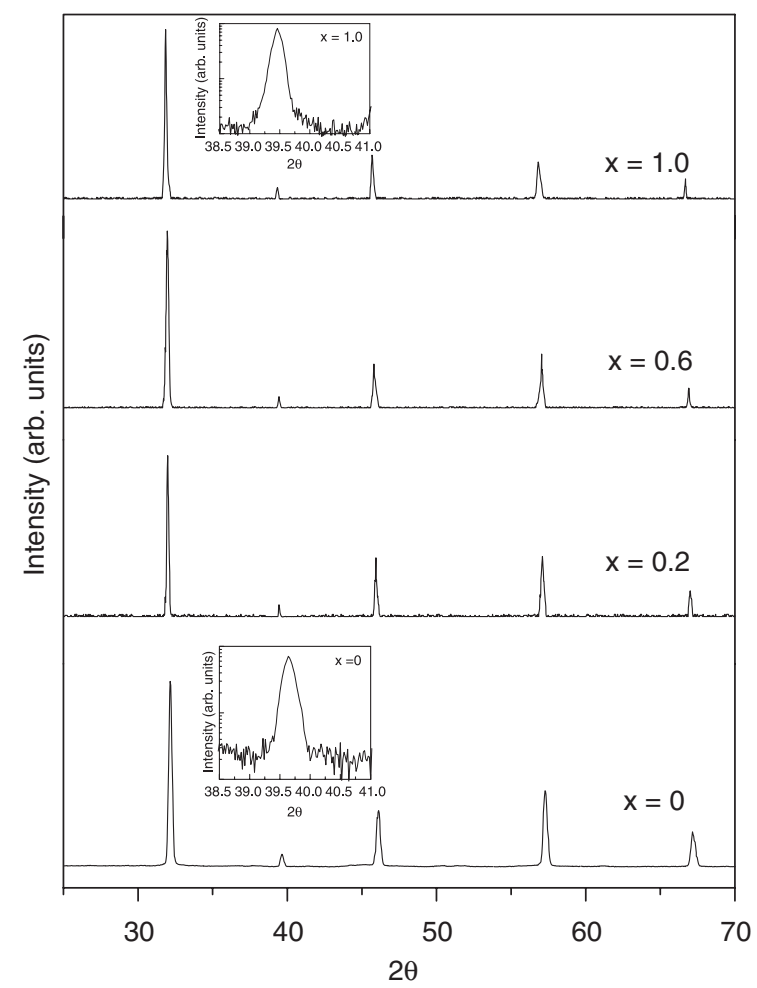

Fig. 1. X-ray diffraction patterns for $\mathrm{Sr}_{2-x} \mathrm{La}_{x} \mathrm{FeMoO}_{6}(x=0$, $0.2,0.6$ and 1.0). The insets show the extended reflection around $39.5^{\circ}$ for $x=0$ and 1.0 samples.

log-normal distribution function given by

$$
\begin{aligned}
\Delta n= & (1 / \sqrt{2 \pi} \ln \sigma) \\
& \times \exp \left\{-\frac{1}{2}[\ln (s / \bar{s}) / \ln \sigma]^{2}\right\} \Delta(\ln s),
\end{aligned}
$$

where $\Delta n$ is the fractional number of grains, $s$ is the grain diameter, $\bar{s}$ is the median diameter and $\sigma$ is the geometric deviation.

The median grain diameters along with geometric deviations for all the samples have been listed in Table 1. It is evident that geometric deviation of each of the median grain diameter is well below the reasonable limit. The median grain diameter increases from $2.16 \mu \mathrm{m}$ for $x=0$ to $2.63 \mu \mathrm{m}$ for $x=0.4$ and then decreases monotonically with higher concentration of $x$ to a value of $0.69 \mu \mathrm{m}$ for $x=1.0$. Addition of La acts as a nucleation center for growing double-layered perovskite crystalline phase, which favors grain growth compared to the pristine oxide at a particular temperature. As we increase La content the number of such nucleation centers increases enormously, which effectively increase the number of crystallites as well as decrease the size and shape anisotropy of crystallites. The variation of median grain size with $\mathrm{La}$ content has been further correlated and explained with magnetotransport properties in latter part of our discussion.

Fig. 4 shows the variation of resistivity with respect to temperature and $\mathrm{La}$ content. The temperature variation of resistivity show semiconducting behavior in all the samples. The resistivity of the undoped sample is higher than La doped samples in whole temperature range, but in doped samples the resistivity increases with increasing $\mathrm{La}$ content. Application of magnetic field reduces the resistivity over the whole temperature range, in particular, the magnitude of MR ratio in low fields. Figs. 5(a) and (b) show the MR ratio with respect to magnetic field $(-0.88-+0.88 \mathrm{~T})$ for all samples at room temperature as well as $10 \mathrm{~K}$, respectively. We define the magnitude of MR as

$\operatorname{MR}(T, H)=[\rho(T, 0)-\rho(T, H)] / \rho(T, 0)$,

where $\rho(0)$ is zero-field resistivity and $\rho(H)$ is the resistivity under magnetic field. At room temperature the MR ratio increases initially up to $x=0.4$ then decreases with increasing La content; while at $10 \mathrm{~K}$ the MR ratio increases initially up to $x=0.2$ then decreases as $x$ increases (see Table 1). Reasonably high MR ratios of $5 \%$ and $7 \%$ with a considerable low magnetic field $(0.88 \mathrm{~T})$ have been obtained in $x=0.2$ and 0.4 samples, respectively, at room temperature. At $10 \mathrm{~K}$, the magnitude of magnetoresistance with magnetic field of $0.88 \mathrm{~T}$ for $x=0.2$ and 0.4 are as large as $13 \%$ and $10 \%$ respectively. In the case of intergranular tunneling magnetoresistance (IMR), two casesintrinsic (i.e. high-field magnetoresistance) and extrinsic (i.e. low-field magnetoresistance), should be distinguished from each other. In low-field extrinsic tunneling magnetoresistance the neighboring grains with thin insulating potential barrier become parallel (magnetically) with the application of small field, which produces a steep rise in MR ratio. The increase of La content increases the mis-site defect concentration [20,21] (which has 


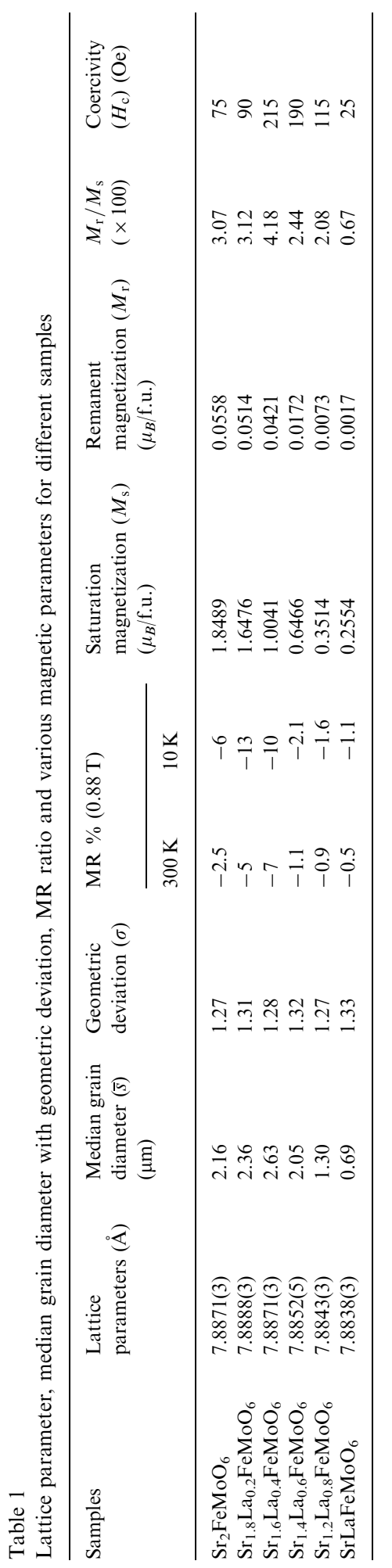



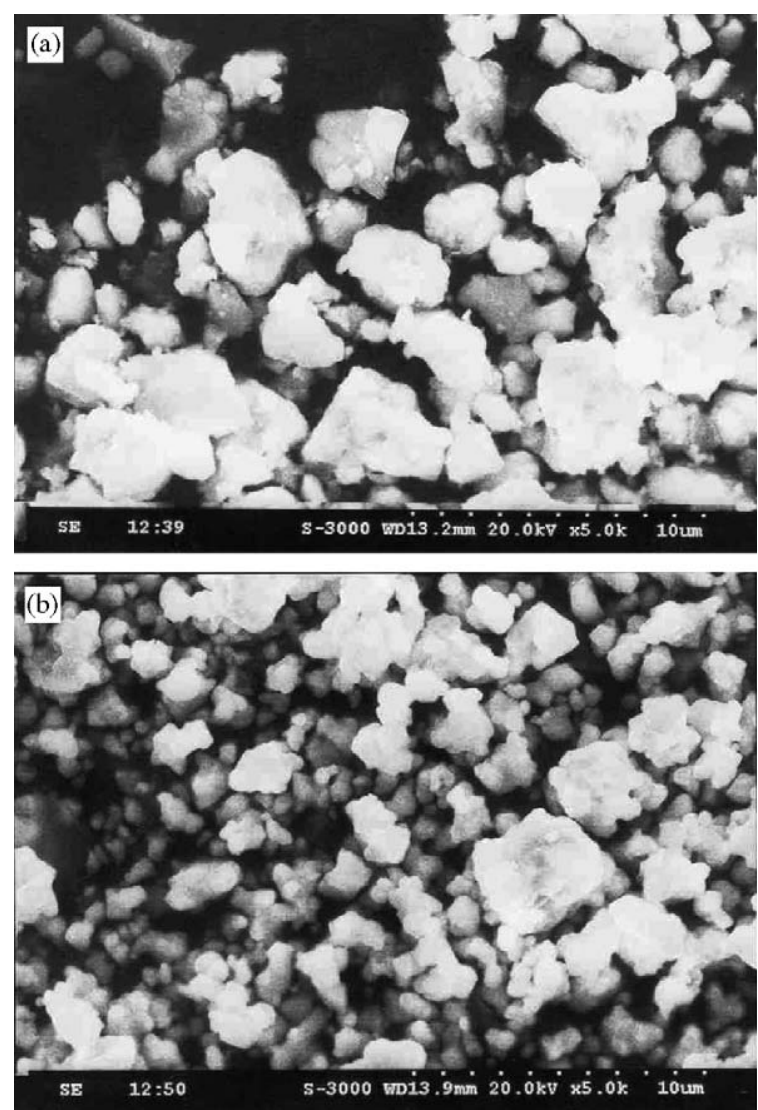

Fig. 2. Scanning electron micrographs of (a) $\mathrm{Sr}_{1.6} \mathrm{La}_{0.4} \mathrm{FeMoO}_{6}$ and (b) $\mathrm{SrLaFeMoO}_{6}$.

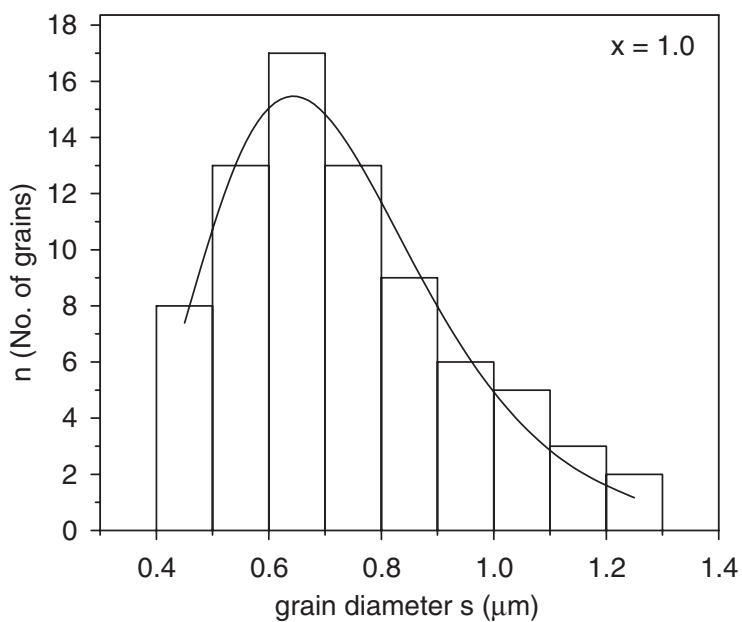

Fig. 3. Histogram of no. of grains vs. grain diameter for $\mathrm{SrLaFeMoO}_{6}$. Solid curve represents the fitting of lognormal distribution function.

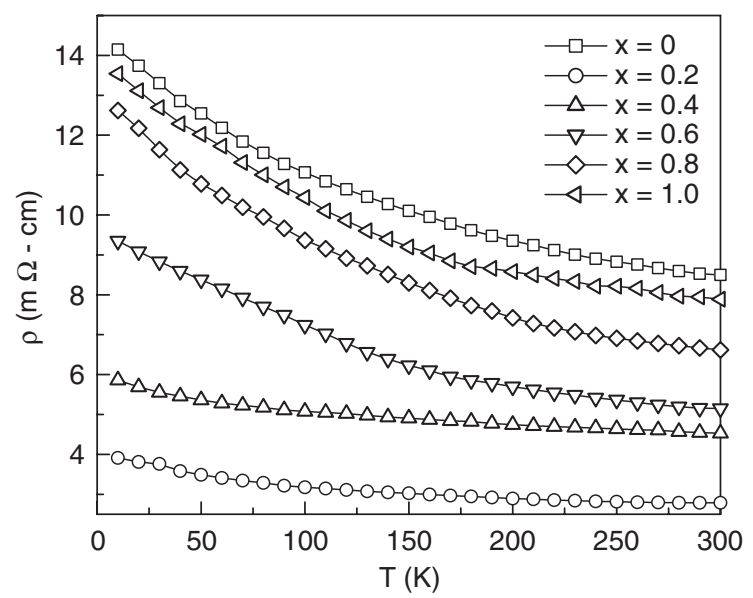

Fig. 4. Temperature dependent resistivity of samples $\mathrm{Sr}_{2-x} \mathrm{La}_{x} \mathrm{FeMoO}_{6}$.
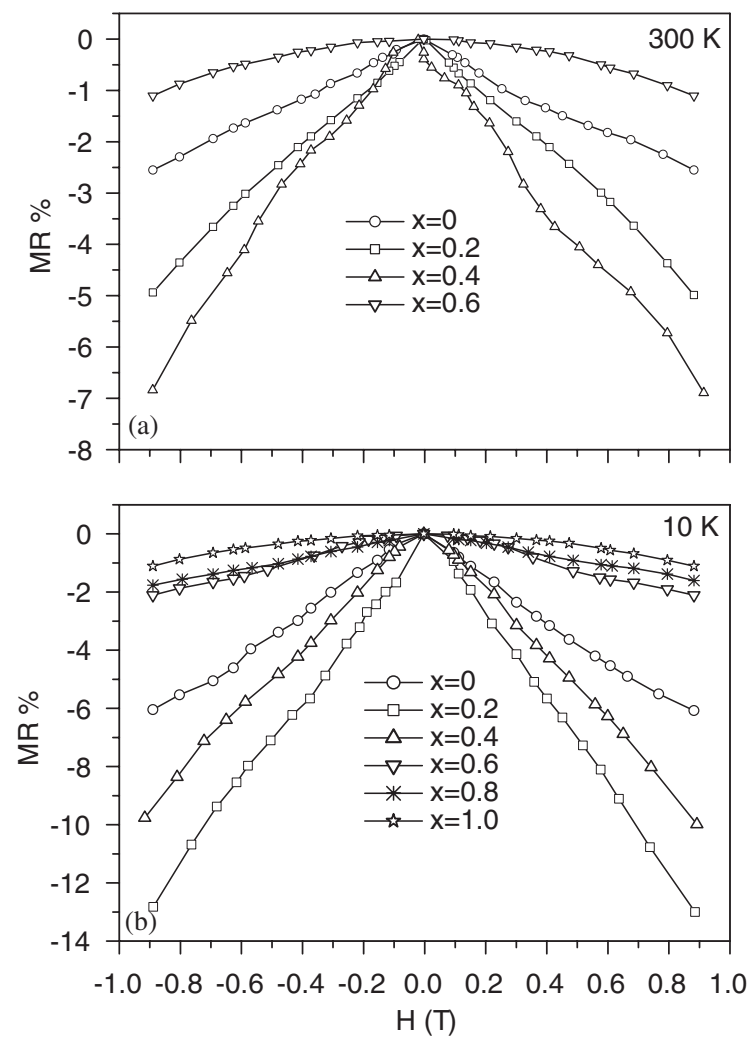

Fig. 5. Magnetoresistance vs. magnetic field for different samples (a) at room temperature and (b) at $10 \mathrm{~K}$. 
been further substantiated in latter part of our discussion), effectively increasing the barrier height, which facilitates the tunneling up to a certain limit of barrier height and hinders the same beyond that. In our case, the limiting value for barrier heights at room temperature and $10 \mathrm{~K}$ are $x=0.4$ and 0.2 , respectively.

Fig. 6 shows the $M-H$ loops taken at $10 \mathrm{~K}$ for all the samples. The saturation magnetization $\left(M_{\mathrm{s}}\right)$ decreases linearly with increasing La, as shown in the inset of Fig. 6. For $x=0$ and 0.2, a high magnetic field $(5 \mathrm{~T})$ is required for saturation, whereas low magnetic fields $(1-2 \mathrm{~T})$ are required for saturating the samples with higher La content. Fig. 7(a) shows variation of remanent magnetization $\left(M_{\mathrm{r}}\right)$ and percentage change of the $M_{\mathrm{r}}$ with respect to $M_{\mathrm{s}}$, Fig. 7(b) shows grain size and coercivity $\left(H_{\mathrm{c}}\right)$ with La content at $10 \mathrm{~K}$, respectively. From the figures we find that $M_{\mathrm{r}}$ decreases as we increases the La content, but the ratio of the $M_{\mathrm{r}}$ with respect to $M_{\mathrm{s}}$, grain diameter, and coercivity increase initially up to $x=0.4$ then decrease with further increase of La content. The magnetic properties of this system at $10 \mathrm{~K}$ are summarized in Table 1.

In one of the earlier Ref. [23], it has been shown that the spin dependent scattering is more suppressed in low fields for samples with smaller grain

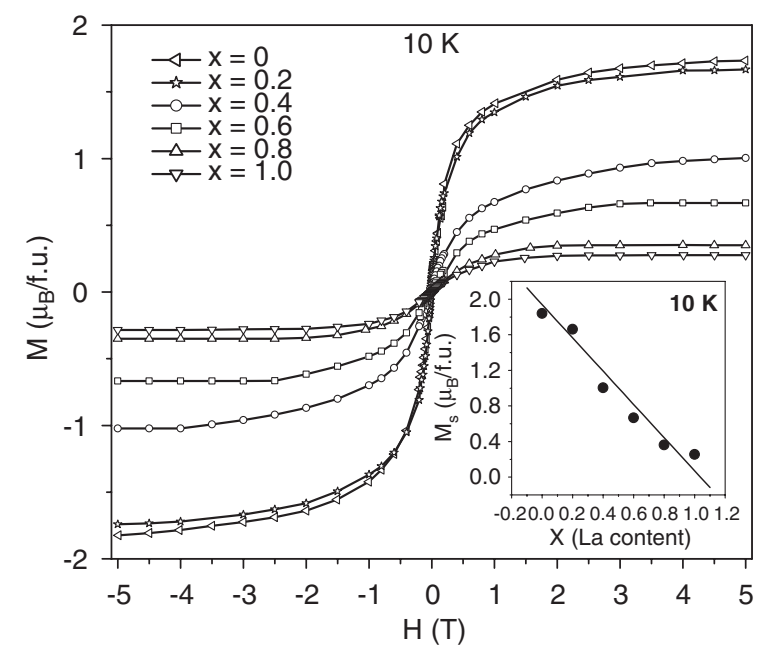

Fig. 6. M-H curves for different samples measured at $10 \mathrm{~K}$. Inset is the variation of saturation magnetization $\left(M_{\mathrm{s}}\right)$ with $\mathrm{La}$ content at $10 \mathrm{~K}$. Solid line represents linear fitting of the data.

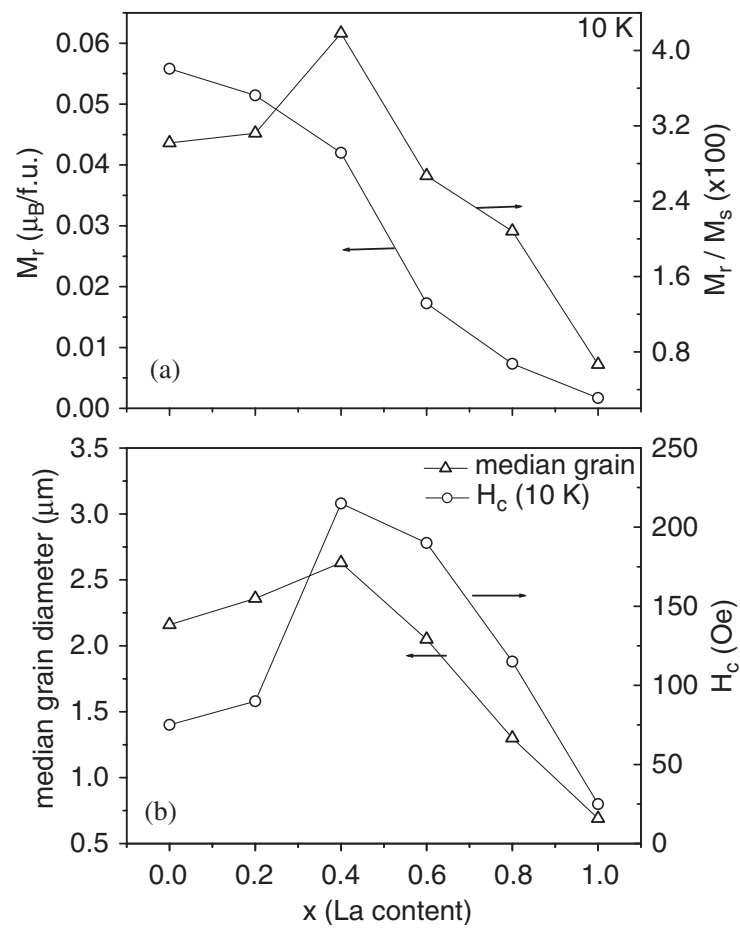

Fig. 7. (a) Variation of remanent magnetization $\left(M_{\mathrm{r}}\right)$ and $\%$ $M_{\mathrm{r}} / M_{\mathrm{s}}$ with La content at $10 \mathrm{~K}$, (b) Variation of median grain diameter and coercivity $\left(H_{\mathrm{c}}\right)$ with La content at $10 \mathrm{~K}$.

size, i.e. for smaller magnetic domains. This will lead to high magnetoresistance ratio at low fields. However, the estimated grain size for such kind of behavior was of the order of nano-meter, which is much smaller than the grain size for the samples in present study. Hence we believe that spin dependent scattering suppression with lowering of grain size is not the predominant effect in present study. On the other hand, analysis of NMR spectrum [24] indicates that the La doping promotes additional electrons into the conducting spin-down band of $\mathrm{Sr}_{2} \mathrm{FeMoO}_{6}$, reflecting the enhancement of the magnetic moment of Mo ions. If more spin-down electrons present inside the grains, the ferromagnetic exchange path of $\mathrm{O}-\mathrm{Fe}-\mathrm{O}-\mathrm{Mo}-\mathrm{O}$ will be disturbed due to disorder of $\mathrm{Fe}$ and Mo atoms at $\mathrm{B}$ site (mis-site defect concentration). Mis-site defect concentration increases with increasing La content $[20,21]$, which leads to the decrease of saturation magnetization $\left(M_{\mathrm{s}}\right)$ as observed in present system 
(shown in the inset of Fig. 6). Monte Carlo simulation [25] also shows that the saturation magnetic moment drops linearly with the mis-site defect concentration for the case of randomly created defects.

The theoretically calculated saturation magnetization for $\mathrm{Sr}_{2} \mathrm{FeMoO}_{6}$ always has a value higher than that of experimentally observed values. Initially by Monte Carlo simulation [25] studies the reduction in magnetic moment has been attributed to random disorder of spin orientation of $\mathrm{Fe}$ and Mo atoms (termed as mis-site defect concentration), where the growth of strong $\mathrm{Fe}-\mathrm{O}-\mathrm{Fe}$ antiferromagnetic coupling diminishes the contribution of $\mathrm{Fe}$ in total magnetic moment. Later the growth of antiphase boundary model has been brought in to account for the lower values of remanent magnetization and saturation field [26]. The anti-phase boundary was evidenced in Morich $\mathrm{Sr}_{2} \mathrm{FeMoO}_{6}$ samples by high resolution transmission electron microscope (HRTEM) [27]. The existence of the antisite antiferromagnetic patches has been established by Mossbauer spectroscopy [28] and is related to the mixed valence state of Fe. Neutron diffraction studies have also revealed reduced spin values at both the $\mathrm{Fe}$ and Mo sites [29], which support the mixed valence states of $\mathrm{Fe}$. Additional support can also be found in magnetization data [25]. The antiferromagnetic patches act as a barrier to the electronic transport between the ordered volumes.

As shown in Fig. 7(b), the coercivity increases from 75 Oe for $x=0$ to a value of 215 Oe for $x=$ 0.4 and then decreases monotonically to 25 Oe for $x=1.0$. The increase in $H_{\mathrm{c}}$ for $x \leqslant 0.4$ can be explained by the reduction in cancellation of spins due to high random disorder within each domain, which is further supported by the relatively higher remanent magnetization and the requirement of very high saturation field $(5 \mathrm{~T})$. The ratio of remanent magnetization with respect to saturation magnetization $\left(M_{\mathrm{r}} / M_{\mathrm{s}}\right)$ increases initially (up to $x=0.4)$ and then decreases to a relatively low value for $x=1.0$ (Fig. 7(a)). Initial increment in the ratio of $M_{\mathrm{r}} / M_{\mathrm{s}}$ and drop in $M_{\mathrm{s}}$ clearly signify that the magnetic interactions are governed by the increase in mis-site defect concentration upon substitution of La for $x \leqslant 0.4$. The rapid drop in ratio of $M_{\mathrm{r}} / M_{\mathrm{s}}$ and $M_{\mathrm{r}}$ coupled with drop in saturating field $(\sim 1 \mathrm{~T})$ and coercivity $\left(H_{\mathrm{c}}\right)$, signify the growth of ferromagnetic ordered domains, which are coupled antiferromagnetically across antiphase boundaries for samples with $x>0.4$. With increasing La content $x$, the decrement in volume mis-match of two different domains across the antiphase boundary will cause the gradual decrease in residual remanent magnetization. This can be further substantiated from the decrease in the grain size and shape anisotropy with increase in La content, as well as the soft magnetic behavior indicated from the $M-H$ curve for sample $x=1.0$. The above observations suggests that a crossover from random disorder of spin orientation to the growth of antiphase boundary in $\mathrm{Sr}_{2-x} \mathrm{La}_{x} \mathrm{Fe}$ $\mathrm{MoO}_{6}$. The random disorder of spin orientation prevails up to $x=0.4$, whereas the gradual growth of antiphase interface is understood to govern the magnetic properties for higher concentration of La.

\section{Conclusions}

Effect of La doping on magnetotransport and magnetic properties has been investigated in double perovskite $\mathrm{Sr}_{2} \mathrm{FeMoO}_{6}$. The $\rho(T)$ curves exhibit characteristics of a semiconductor behavior for all the samples. At $0.88 \mathrm{~T}$, the MR ratios of $5 \%$ $(13 \%)$, and $7 \%(10 \%)$ at room temperature $(10 \mathrm{~K})$ have been obtained for samples $x=0.2$ and 0.4 , respectively. The observed increase of $H_{\mathrm{c}}, M_{\mathrm{r}} / M_{\mathrm{s}}$, high saturation fields, and reduction of the saturation magnetization for $x \leqslant 0.4$ indicate that random disorder of spin orientation is mainly responsible for the enhancement of low-field MR. For $x>0.4$, rapid drop of $H_{\mathrm{c}}, M_{\mathrm{r}} / M_{\mathrm{s}}, M_{\mathrm{r}}$, and saturation fields cause the growth of antiphase boundary and give rise to lower values of low-field magnetoresistance.

\section{Acknowledgements}

This work has been supported by National Science Council of ROC under Grant No. NSC 90-2112-M-002-042. 


\section{References}

[1] S. Jin, T.H. Tiefel, M. McCormack, R.A. Fastnacht, R. Ramesh, H.L. Chen, Science 264 (1994) 413.

[2] M. Imada, A. Fujimori, Y. Tokura, Rev. Mod. Phys. 70 (1998) 1039.

[3] C. Zener, Phys. Rev. 82 (1951) 403.

[4] K.-I. Kobayashi, T. Kimura, H. Sawada, K. Terakura, Y. Tokura, Nature (London) 395 (1998) 677.

[5] K.-I. Kobayashi, T. Okuda, Y. Tomioka, T. Kimura, Y. Tokura, J. Magn. Magn. Mater. 218 (2000) 17.

[6] F.K. Patterson, C.W. Moeller, R. Ward, Inorg. Chem. 2 (1963) 196.

[7] W. Westerburg, D. Reisinger, G. Jakob, Phys. Rev. B 62 (2000) R767.

[8] Y. Moritomo, Sh. Xu, A. Machida, T. Akimoto, E. Nishibori, M. Takata, M. Sakata, Phys. Rev. B 61 (2000) R7827.

[9] J.P. Zhou, R. Dass, H.Q. Yin, J.-S. Zhou, L. Rabenberg, J.B. Goodenough, J. Appl. Phys. 87 (2000) 5037.

[10] F.S. Galasso, F.C. Douglas, R.J. Kasper, J. Chem. Phys. 44 (1966) 1672.

[11] Y. Tomioka, T. Okuda, Y. Okimoto, R. Kumai, K.-I. Kobayashi, Y. Tokura, Phys. Rev. B 61 (2000) 422.

[12] F.S. Galasso, in: R. Smoluchouski, N. Kurti (Eds.), In International Series of Monographs in Solid State Physics, Pergamon, London, 1969.

[13] M. Itoh, I. Ohta, Y. Inaguma, Mater. Sci. Eng. B 41 (1996) 55.

[14] Yu. Lu, X.W. Li, G.Q. Gong, G. Xiao, A. Gupta, P. Lecoeur, J.Z. Sun, Y.Y. Wang, V.P. Dravid, Phys. Rev. B 54 (1996) R8357.
[15] W. Westerburg, F. Martin, S. Friedrich, M. Mair, G. Jakob, J. Appl. Phys. 86 (1999) 2173.

[16] M. Viret, M. Drouet, J. Nassar, J.P. Contour, C. Fermon, A. Fert, Europhys. Lett. 39 (1997) 545.

[17] Phys. Today April (1995), Special Issue: Magnetoelectronics.

[18] K.-I. Kobayashi, T. Kimura, H. Sawada, K. Terakura, Y. Tokura, Phys. Rev. B 59 (1999) 11159.

[19] Y. Moritomo, S. Xu, T. Akimoto, A. Machida, N. Hamada, K. Ohoyama, E. Nishibori, M. Takata, M. Sakata, Phys. Rev. B 62 (2000) 14224.

[20] J. Navarro, C. Frontera, L1. Balcells, B. Martinez, J. Fontcuberta, Phys. Rev. B 64 (2001) 92411.

[21] M. Garcia-Hernandez, J.L. Martinez, M.J. MartinezLope, M.T. Casais, J.A. Alonso, Phys. Rev. Lett. 86 (2001) 2443

[22] J. Navarro, J. Nogues, J.S. Munoz, J. Fontcuberta, Phys. Rev. B 67 (2003) 174416.

[23] C.L. Yuan, S.G. Wang, W.H. Song, T. Yu, J.M. Dai, S.L. Ye, Y.P. Sun, Appl. Phys. Lett. 75 (1999) 3853.

[24] M. Wojcik, E. Jedryka, S. Nadolski, J. Navarro, D. Rubi, J. Fontucuberta, Phys. Rev. B 69 (2004) 100407.

[25] A.S. Ogale, S.B. Ogale, R. Ramesh, T. Venkatesan, Appl. Phys. Lett. 75 (1999) 537.

[26] J.B. Goodenough, R.I. Dass, Int. J. Inorg. Mat. 2 (2000) 3.

[27] T.T. Fang, Phys. Rev. B 71 (2005) 64401.

[28] J. Linden, T. Yamamoto, M. Karppinen, H. Yamauchi, Appl. Phys. Lett. 76 (2000) 2925.

[29] B. Garcia Landa, C. Ritter, M.R. Ibarra, J. Blasco, P.A. Algarabel, R. Mahendiran, J. Garcia, Solid State Comm. 110 (1999) 435. 